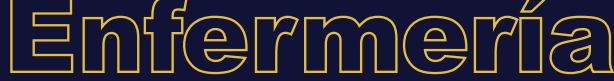 \\ Unìgrsitarĩa
}

\section{Transitando hacia el ejercicio de autonomía y liderazgo: ... «para el posicionamiento hay que luchan»}

\section{Moving towards the practice of autonomy and leadership:... "Positioning requires striving»}

\section{Transitar para o exercício de autonomia e liderança: ... «Para o posicionamento, tem que lutar»}

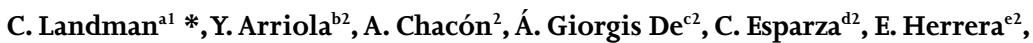
P. Pizarro ${ }^{2}$, S. Ribera ${ }^{\mathrm{f} 2}$, D. Rojas ${ }^{\mathrm{g} 2}$, V. Verdejo ${ }^{\mathrm{h} 2}$, J. Molina ${ }^{\mathrm{i} 2}$

ORCID:

$\begin{array}{lll}{ }^{\mathrm{a}} 0000-0002-3318-0971 & { }^{\mathrm{b}} 0000-0001-5423-015 \mathrm{X} & { }^{\mathrm{c}} 0000-0003-2466-4904 \\ { }^{\mathrm{d}} 0000-0002-0707-8922 & { }^{\text {e }} 0000-0003-4301-9281 & { }^{\mathrm{f}} 0000-0002-9146-207 \mathrm{X}\end{array}$

${ }^{\mathrm{g}} 0000-0003-2092-7607 \quad{ }^{\mathrm{h}} 0000-0002-8311-9896 \quad{ }^{\mathrm{i}} 0000-0002-9614-2841$

${ }^{1}$ Departamento de Enfermería Adulto y Adulto Mayor, Escuela de Enfermería, Facultad de Medicina, Universidad de Valparaíso, Valparaíso, Chile

${ }^{2}$ Escuela de Enfermería, Facultad de Medicina, Universidad de Valparaíso, Valparaíso, Chile

Recibido: 30 marzo 2018

Aceptado: 15 enero 2019

\section{Resumen}

Introducción: En Chile, las enfermeras/os tienen respaldo legal para gestionar el cuidado, esto facilita el desarrollo de competencias para ejercer autonomía y liderazgo. Actualmente persisten barreras para transitar hacia un reconocimiento real de la independencia profesional.

Objetivo: Develar la experiencia vivida de enfermeras con cargos en niveles de jefatura respecto al propio desarrollo para lograr el liderazgo y autonomía profesional.

Metodología: Estudio cualitativo, descriptivo-interpretativo, desde la trayectoria fenomenológica de Edmund Husserl. Muestreo selectivo de caso homogéneo de tres enfermeras que desempeñaban rol de jefatura en instituciones de salud, región Valparaíso, Chile. Participación consentida. Entrevista no estructurada. Análisis del discurso utilizando el modelo de liderazgo integral de Ken Wilber. 
Resultados: Categorías: Conciencia del líder «ser inquieta...intelectualmente». Construyendo competencias «el liderazgo se aprende». Transitando hacia el ejercicio de la autonomía y el liderazgo, "para el posicionamiento hay que luchar”. El peso de las estructuras organizacionales «tu rol es parte de un hospital». Interpretación: El ejercicio del liderazgo y la autonomía se fundamenta en competencias actitudinales por sobre las cognitivas y técnicas. Existe mayor apoyo y reconocimiento a nivel institucional, que al interior del equipo de salud. Persisten limitaciones para lograr máxima expresión del liderazgo y autonomía, por persistencia de modelos institucionales hegemónicos, centrados en decisiones médicas y desconocimiento del rol integrado.

Conclusión: El líder se mueve en un escenario dual, entre un menor reconocimiento del equipo y un creciente empoderamiento del rol autónomo y visibilidad a nivel directivo institucional.

Palabras clave: Autonomía profesional; liderazgo; rol profesional; rol de la enfermera; enfermeras/os; supervisión de enfermería; Chile.

\section{Abstract}

Introduction: In Chile, nurses have legal support to manage care, a situation which facilitates the development of competencies to perform with autonomy and leadership; however, barriers still persist while moving towards a real acknowledgement of professional independency.

Objective: To unveil the lived experience of nurses in executive roles regarding their own development towards leadership and professional autonomy.

Methodology: This is a descriptive and interpretative qualitative study framed by the Edmund Husserl phenomenological approach. The sample by homogeneous case included three nurses in executive roles in health institutions in Valparaiso, Chile. The participation was by consent. An unstructured interview was used. For the discourse analysis, the Ken Wilber integral leadership model was followed.

Results: Categories: Leadership awareness < being inquiring... intellectually $>\ldots$ Building Competencies <leadership is learned $>$. Moving towards the practice of autonomy and leadership, "positioning requires striving”. Weight of the organizational structures <your role is part of a hospital>. Interpretation: A practice of leadership and autonomy is based on attitude competencies over the cognitive and technical ones. Nevertheless, there still persist limitations to achieve a maximal expression of leadership and autonomy due to hegemonic institutional models which focus on medical decisions and neglect the integrated roles.

Conclusion: The leader moves in a dual setting between a lower acknowledgement from the team, and a higher empowerment of the autonomous role and the visibility at the institutional directive level.

Keywords: Professional autonomy; leadership; professional role; nurse role; nurses; nursing, supervisory; Chile.

\section{Resumo}

Introdução: No Chile, as enfermeiras/os têm apoio legal para gerenciar o cuidado, isto facilita o desenvolvimento de competências para exercer autonomia e liderança. Atualmente, persistem barreiras para transitar em direção a um reconhecimento real da independência profissional.

Objetivo: Desvendar a experiência vivida de enfermeiras com cargos em níveis de chefia ao respeito do próprio desenvolvimento, para atingir a liderança e autonomia profissional.

Metodologia: Estudo qualitativo, descritivo-interpretativo, desde a trajetória fenomenológica de Edmund Husserl. Amostragem seletiva de caso homogéneo de três enfermeiras que desempenhavam papel de chefia em instituições de saúde, região Valparaíso, Chile. Participação consentida. Entrevista não estruturada. Análise de discurso, utilizou-se o modelo de liderança integral de Ken Wilber.

Resultados: Categorias: Consciência do líder «ser irrequieta...intelectualmente». Ir construindo competências «a liderança se aprende». Transitar em direção ao exercício da autonomia e a liderança, "para o posicionamento tem que lutar”. O peso das estruturas organizacionais «seu rolo é parte de um hospital». Interpretação: O Exercício da liderança e a autonomia fundamenta-se em competências atitudinais, sobre as cognitivas e técnicas. 
Existe maior apoio e reconhecimento a nível institucional, que no interior da equipe de saúde. Persistem limitações para atingir a máxima expressão de liderança e autonomia, por persistência de modelos institucionais hegemónicos, centrados em decisões médicas e desconhecimento do rolo integrado.

Conclusão: O líder movimenta-se em um cenário dual, entre um menor reconhecimento da equipe e um empoderamento crescente do rolo autónomo e visibilidade a nível diretivo institucional.

Palavras chave: Autonomia profissional; liderança; rolo profissional; rolo da enfermeira; enfermeiras/os; supervisão de enfermagem; Chile.

\section{Introducción}

El desarrollo de la enfermería como profesión y disciplina ha evolucionado a través del tiempo, fundamentalmente, hacia el posicionamiento del rol de líder autónomo al interior de los equipos de salud ${ }^{1}$. Se ha realizado un largo camino desde los cuidados domésticos, vocacionales, técnicos, hasta alcanzar la impronta profesional ${ }^{2,3}$. Letelier y Velandia señalan que se debiera avanzar aún más, desde la profesionalización al profesionalismo, como una apuesta de integración teórica y práctica, en donde se combine conocimiento, con saber hacer y saber moral, que se facilite el aprendizaje para la toma de decisiones de manera autónoma en la acción del cuidar y no sólo cumplir con indicaciones médicas y realizaciones de carácter técnico, esto es, asumir el liderazgo para la gestión del cuidado en su integridad ${ }^{1,4}$.

El fundamento para realizar esta investigación es a partir de la experiencia de los autores del estudio, quienes durante las prácticas clínicas realizadas en diversos centros asistenciales, han observado dificultades para que las enfermeras/os puedan insertarse como líderes reconocidos al interior de los equipos de salud y que se respete su autonomía decisional.

En Chile, actualmente, las enfermeras y enfermeros cuentan con el respaldado legal para asumir la gestión del cuidado de manera autónoma, privilegio logrado tras prolongadas luchas gremiales, transformándose en una de las bases en que se fundamenta el ejercicio del liderazgo y la autonomía profesional. El marco legal, que sustenta el ejercicio profesional, se expresa en la Ley de Autoridad Sanitaria 19.536, de 1997, articulo 113 y en la Norma General Administrativa № 19, del año 2007. El primero define el rol profesional y la segunda operacionaliza el Modelo de Gestión del Cuidado de Enfermería, responsabilidad de las enfermeras/os. En el nivel directivo institucional ambos se representan en atribuciones para organizar, supervisar, evaluar y promover el mejoramiento de la calidad de los cuidados de enfermería, a fin de otorgar una atención segura, oportuna, continua, con pertinencia cultural, acorde con las políticas y normas ministeriales ${ }^{5-7}$.

Si bien es cierto, en este último tiempo se ha progresado para el logro de la autonomía y el ejercicio del liderazgo en enfermería, aún no se ha llegado al destino, debido a la persistencia de barreras que limitan el reconocimiento del rol por parte de algunos integrantes del equipo de salud y de los usuarios de los centros sanitarios. Algunas de las limitaciones observadas, se producen por la presencia hegemónica del modelo biomédico al interior de las instituciones, lo que genera niveles de subordinación que desdibujan la imagen del profesional ${ }^{1,8-12}$.

En este escenario dual, de respaldo legal por un lado y de menor reconocimiento social por otro, las enfermeras/os muestran diversos niveles de autonomía según el área de desempeño del rol, por ejemplo: se observa una mayor independencia en las funciones derivadas de la asistencia, especialmente en lo referente a la educación y a la promoción en salud, en contrapartida con un menor grado de independencia en las funciones derivadas de la gestión e investigación. Esta situación se ve agravada por la escasa participación de dichos profesionales en las organizaciones gremiales, lo cual limita una defensa colectiva para el reconocimiento social del rol profesional ${ }^{10}$.

Los conceptos de autonomía y liderazgo en enfermería asumidos en este estudio, son considerados como una entidad diádica en el área de la gestión y administración, ya que ambos se potencian entre sí en una amalgama simbólica, para lograr el empoderamiento y el necesario reconocimiento de los profesionales de enfermería, en un ejercicio de liderazgo participativo, basado en la confianza e 
interdependencia entre los miembros del equipo de salud, con lo que se logra así, un mayor grado de satisfacción y compromiso con la institución y el trabajo desempeñado ${ }^{1,13-17}$.

Diversos estudios consideran a la autonomía, como uno de los atributos e indicadores que definen al profesionalismo de la enfermera/o, ya que permite su propio posicionamiento en el equipo de salud y condiciona el empoderamiento en el ejercicio de su rol, en cuanto a manifestar independencia en la práctica, como en la aplicación del juicio crítico para tomar decisiones, implica correr riesgos, responsabilizarse de los propios actos, tener la capacidad de autodeterminación y de autorregulación $n^{4,8,18,19}$.

Al liderazgo se le relaciona directamente con el ejercicio autónomo de la profesión, y se entiende como un fenómeno colectivo que implica un proceso de influencia de una persona sobre los demás, con el fin de lograr metas comunes, tanto institucionales como de gestión del cuidado, con énfasis en la eficacia y en la entrega de un servicio profesional de alta calidad ${ }^{18-20}$. Peter Druker agrega que el liderazgo efectivo surge del compromiso de servir a los demás, en vez de a uno mismo, lo cual fortalece de manera colaborativa a las organizaciones en las que se labora ${ }^{21}$.

Los antecedentes revisados brindan fundamento para la realización de este estudio con un enfoque cualitativo descriptivo-interpretativo, desde la trayectoria fenomenológica de Edmund Husserl, ya que en gran medida, las investigaciones en el área del liderazgo en enfermería corresponden al paradigma cuantitativo con orientación hacia las teorías psicológicas y organizacionales, que subyacen tras los estilos de liderazgo desarrollados al interior de los equipos de salud ${ }^{22-26}$, también, se observa una menor presencia de estudios con abordaje cualitativo y de reflexión, a partir de las experiencias de enfermeras que ejercen el liderazgo ${ }^{18,19,27}$.

Como una manera de acercarse a la comprensión del fenómeno estudiado se utilizó el modelo AQAL (All Quadrants, All Levels) del psicólogo Ken Wilber, quien analiza los diferentes niveles comprensivos del llamado liderazgo integral, el cual se aplicaría, según el autor, a cualquier organización, no exclusivamente a instituciones de salud ${ }^{28}$.

El modelo de Ken Wilber define al liderazgo integral como la capacidad de acoger todas las áreas de interacción de las personas, sean estás internas y externas, individuales y colectivas, permitiéndole generar consciencia, motivación y pasión a los miembros de un grupo, así como interconectar e interrelacionar los procesos de cambio que se dan al interior de las instituciones ${ }^{28}$.

Para develar la propia construcción del liderazgo y de la autonomía profesional de enfermeras/os que ejercen cargos de responsabilidad en la gestión, se realizó una inmersión en el mundo personal subjetivo como reflejo de la realidad intersubjetiva de este colectivo $^{29}$, a través de la siguiente pregunta investigativa: ¿Cuál es la experiencia vivida de enfermeras que ejercen cargos de jefaturas respecto al propio desarrollo del liderazgo y de la autonomía profesional? Como objetivo general del estudio se pretende develar la experiencia vivida en enfermeras con cargos en niveles de jefatura respecto al propio desarrollo, para lograr el liderazgo y la autonomía profesional. Los objetivos específicos son identificar los valores y las competencias de las enfermeras que ejercen cargos de jefaturas. Junto con ello, identificar las facilidades y las barreras para desarrollar cargos de liderazgo y ejercer la autonomía, desde las vivencias de estas enfermeras.

\section{Material y método}

Estudio de diseño cualitativo, descriptivo e interpretativo, desde la fenomenología de Edmund Husserl, para develar la experiencia vivida de enfermeras con cargos en niveles de jefatura, representadas en fragmentos de la conciencia que es necesario describir para acercarse a la comprensión del fenómeno de estudio ${ }^{29}$. La fundamentación de la selección del método fenomenológico utilizado, se basa en el reconocimiento que se les asigna a las vivencias intencionales relatadas desde el yo, así como a la rememoración de estas, como fuente de información para iniciar la reflexión acerca de la construcción de las competencias personales del líder y el desarrollo de la autonomía, desde el espacio temporal y el contexto institucional de las enfermeras entrevistadas ${ }^{29}$. 
Se realizó la selección de los participantes, a través de un muestreo selectivo de caso homogéneo de tres enfermeras que desempeñaban roles de jefatura. Los criterios de selección muestral consideraron ser enfermera o enfermera-matrona; pertenecer a tres generaciones etarias diferentes; ejercicio profesional por un tiempo igual o mayor a 15 años; ejercer cargos de jefatura por cinco años o más; pertenecer a centros de salud de atención cerrada, complejos, públicos o privados; tener formación de postítulo en áreas afines a la gestión en salud. Los criterios de inclusión se fundamentaron en la posibilidad, de que las participantes proporcionaran información en profundidad y detalles suficientes, para develar y comprender el fenómeno de estudio, así como la complejidad de los procesos sociales que lo circundan ${ }^{30}$ (Tabla 1).

Tabla 1. Caracterización de las informantes clave

\begin{tabular}{llll}
\hline $\begin{array}{l}\text { Informantes } \\
\text { claves }\end{array}$ & Formación & Cargo & Locus \\
\hline E1 & $\begin{array}{l}\text { Enfermera-matrona, } \\
\text { Diplomada gestión salud. (c) Magister } \\
\text { Enfermería mención gestión cuidado }\end{array}$ & $\begin{array}{l}\text { Subdirección } \\
\text { enfermería }\end{array}$ & $\begin{array}{l}\text { Centro salud } \\
\text { privado, V región }\end{array}$ \\
\hline E2 & $\begin{array}{l}\text { Enfermera-matrona, } \\
\text { Diplomada gestión salud }\end{array}$ & $\begin{array}{l}\text { Supervisora servicio } \\
\text { de cirugía }\end{array}$ & $\begin{array}{l}\text { Hospital público, tipo A, } \\
\text { V región }\end{array}$ \\
E3 & Enfermera, Diplomada gestión salud & $\begin{array}{l}\text { Dirección unidad } \\
\text { de calidad }\end{array}$ & $\begin{array}{l}\text { Hospital público, tipo A, } \\
\text { V región }\end{array}$ \\
\hline
\end{tabular}

Aspectos éticos: las informantes fueron invitadas a participar sin coacción, enteradas de los objetivos del estudio y del valor que ellas podrían aportar al mismo. Firmaron consentimiento informado, con aceptación de la grabación de las entrevistas. Se respetó el anonimato y la confidencialidad de la información ${ }^{31}$.

Relación entre participantes e investigadores: uno de los investigadores se había relacionado académicamente con las informantes en el pasado, en un estar ahí compartido, lo cual facilitó el proceso de triangulación. Para respetar los criterios de credibilidad, confirmabilidad y dependencia de la investigación, el resto de los investigadores no tenían relación con las participantes, esto como una manera cautelar de que sus perspectivas no interfirieran en la naturaleza de la información obtenida durante las entrevistas ${ }^{31}$.

Procedimiento de recolección de la información: el trabajo de campo se realizó en el espacio laboral de cada informante, previa coordinación y aceptación de las involucradas, entre abril y junio, 2015, en las ciudades de Viña del Mar y Valparaíso.

Se utilizó la entrevista fenomenológica que consistió en tres preguntas abiertas que permitieron que cada enfermera se explayara y diera a conocer sus perspectivas, así como vivencias en relación con el tema. La duración de la entrevista estuvo determinada por la profundidad, nivel de claridad de la información, saturación discursiva y descripciones minuciosas de la información ${ }^{32}$.

\section{Guión de preguntas orientadoras}

1) ¿Cómo cree usted que se desarrolla el liderazgo y la autonomía del profesional de enfermería?

2) ¿Cuál ha sido su trayectoria para llegar a su actual posición?

3) ¿Cuál ha sido su experiencia en el ejercicio de su rol de jefatura?, además de identificar principales facilidades y posibles barreras del contexto relacional e institucional.

Cada entrevista fue grabada y realizada por tres investigadores, uno de ellos mantuvo la atención en el relato y los dos restantes se encargaron de observar el énfasis discursivo, que orientaron a la determinación de las categorías, la información obtenida se registró en un cuaderno de campo. La duración de la entrevista fue entre 50 y 70 minutos, en ese tiempo se logró la saturación de los discursos, es decir cuando la información entregada por las participantes no aportaba nuevos elementos (criterios de confirmabilidad y dependencia) ${ }^{31,33}$. 
Análisis de la información y descripción densa: consideró las fases establecidas por Taylor y Bogdan, descubrimiento, codificación y relativización de los datos, en búsqueda de los significados ${ }^{34}$.

Fase de descubrimiento: Las entrevistas fueron transcritas textualmente, para una posterior lectura repetida por parte de los investigadores. Se buscaron las pistas de temas y posibles tipologías. El equipo de investigadores realizó una discusión para consensuar unidades de significados preliminares que respondieran a la pregunta investigativa ${ }^{34}$. Se realizaron nuevas revisiones bibliográficas con el fin de ir vinculando la información con la teoría, se logró un reordenamiento de las unidades de significado, que develaran los conceptos y proposiciones del fenómeno en estudio. En esta fase, se seleccionó el modelo desarrollado por Ken Wilber, puesto que las categorías encontradas se moldeaban de manera significativa al liderazgo integral propuesto en el modelo ${ }^{28}$.

Fase de codificación: las unidades de significado se codificaron y agruparon en una matriz analítica que consideró las proposiciones argumentativas representativas de una determinada categoría, en el contexto de las características del líder y del desarrollo de la autonomía. Se identificaron cuatro categorías, correspondientes al modelo AQAL de liderazgo integral de Wilber 28,35 .

El modelo de Wilber presenta cuatro dimensiones, organizadas en cuadrantes concatenados entre sí. El superior izquierdo, centrado en la comprensión psicológica del yo interior-individual, hace referencia a procesos correspondientes a la esfera subjetiva o mundo personal fenomenológico; el inferior izquierdo, interior-colectivo, referido al nosotros enfatiza la cultura organizacional, comprende las dimensiones intersubjetivas de las experiencias, como costumbres, rituales, valores, acuerdos compartidos por un colectivo. El cuadrante superior derecho, exterior-individual, corresponde al ello o conductual, se refiere a las dimensiones objetivas o manifiestas del comportamiento individual de la persona y los procesos biológicos, neuroquímicos involucrados en ella. El cuadrante inferior derecho, exterior-colectivo o social, corresponde al ellos en sus dimensiones interobjetivas de la experiencia, comprende la construcción material y visible del mundo, los sistemas sociales y su entorno ${ }^{28}$ (Figura 1).

Fase de relativización de la información: se consideró la interpretación de la información y el proceso de triangulación: (con la teoría, el marco empírico, con otros investigadores e informantes) ${ }^{34}$.

\section{Resultados}

Los resultados presentados corresponden al análisis de los discursos, en oposiciones argumentativas, que describen las propias vivencias de las enfermeras respecto al desarrollo del liderazgo y autonomía profesional.

1. Conciencia del líder «ser inquieta...intelectualmente». Esta categoría quedó constituida por tres dimensiones, valores personales-laborales, gestión, conocimiento e indagación.

Valores personales/laborales

«...el liderazgo no está asignado por un cargo, sino que hay un liderazgo que se da de forma natural en las personas, de que también está motivado, por aspectos personales, de que si tú eres inquieto intelectualmente, si te gusta indagar...» (E1-p15).

«... un buen líder además de tener vocación de servicio (Nota de Inv: énfasis) y de tener habilidades (...) el tema del respeto, la honestidad...porque todas esas características que incluso se establecen como en los valores propios de la institución, tienen que ser los valores propios también del líder» (E3-p7).

Gestión

«...yo puedo decir que este hospital se acreditó gracias al trabajo ciento por ciento de las enfermeras supervisoras de todos los servicios...» (E2-p2).

«... todo lo que es decisiones estratégicas de esta institución pasan en algún momento por cosas de enfermería, por lo tanto el tener línea directa con la parte directiva es necesario...» (E1-p20). 


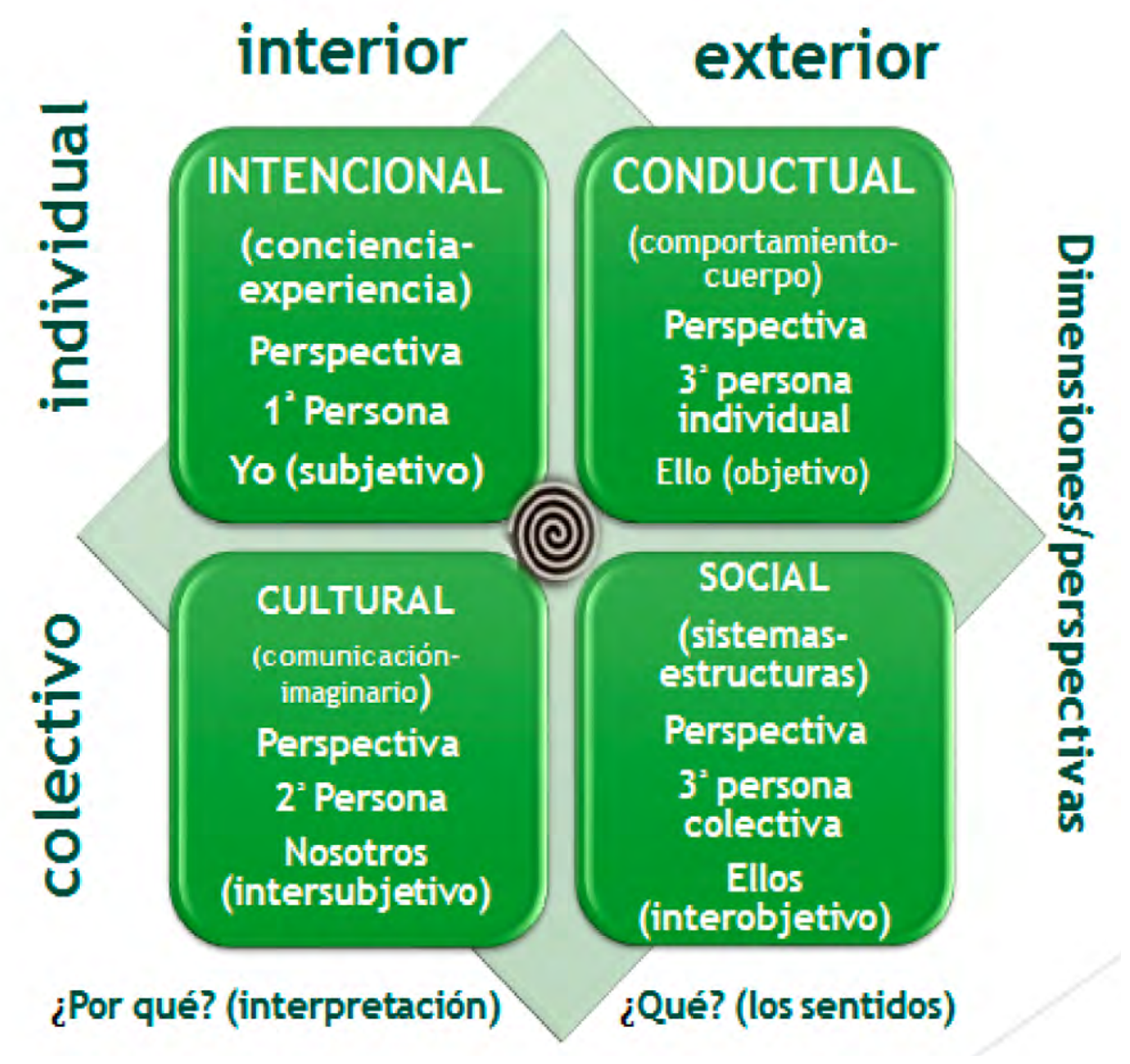

Conocimiento e indagación

«...poder tomar desafíos eh primero ser inquieta intelectualmente para que te dé la seguridad ( $\mathrm{N}$ de I: énfasis) ... que sabes lo que haces, eh lo segundo es siempre querer aprender más cosas no limitarte...el tomar riesgos, el tomar oportunidades...» (E2-p37).

«...yo creo que la autonomía va ligada al conocimiento..., tienes el poder de decisión...y formar al equipo» (E1-p19).

2. Construyendo competencias «el liderazgo se aprende». Las informantes señalaron competencias actitudinales y profesionales. Las primeras son: la comunicación, la escucha activa, ser justas, generar buenas relaciones humanas, considerar al otro sin autoritarismo. En cuanto a las competencias profesionales, se señala un liderazgo participativo, la gestión de recursos humanos y materiales, el manejo en crisis, la experticia clínica, el trabajo en equipo y la resolución de problemas.

Competencias genéricas actitudinales:

«La comunicación con los pares, la habilidad también de poder de resolución de problemas, desarrollar la capacidad de resolver conflictos...» (E2-p17).

«...tienes que saber escuchar, tienen que ser para ti, todas las personas iguales, no tener bando, mostrar a la gente y ser justa...» (E3-p2).

«...las buenas relaciones en la medida que tú puedas ir desarrollando equipos de trabajo...» (E1-p38) 
Competencias profesionales

«...desarrollar la capacidad de resolver conflictos y resolver problemas que se presentan, desde algo técnico, no sé, que te funcione mal un equipo, algo muy técnico, hasta problemas y conflictos de relaciones humanas entre las paramédicos, tuyo con colegas, eh, con los familiares» (E1-p17).

«...ser capaz de equilibrar lo que es el poder que está impuesto de alguna forma eh, con el grupo. O sea el poder, con este cargo ejercer un liderazgo que también sea participativo» (E1-p15).

3.Transitando hacia el ejercicio de la autonomía y el liderazgo, «para el posicionamiento hay que luchar». Las dimensiones encontradas se orientan hacia barreras y facilidades para lograr el reconocimiento del equipo. Las barreras identificadas son las relaciones de poder operantes en la institución, la imagen profesional desvirtuada y el desconocimiento del rol profesional integrado por parte del equipo. Como facilidades se describe la conciencia de las propias capacidades y el reconocimiento legal del rol profesional.

\section{Barreras:}

Relaciones de poder operantes en las instituciones de salud

«...hubo un pronunciamiento del colegio médico en que tenían mucho temor de que (...) ellos hablaban de que la gestión clínica se estaba como dividiendo y que toda la implementación de las subdirecciones de enfermería, lo que iban a hacer iban a dividir la... los equipos de trabajo, más que unirlos...» (E1-p22).

«... se da en los estamentos médicos en que cuesta un poco el aceptar la autonomía de la parte de enfermería...culturalmente en los estamentos médicos queda mucho de eso...» (E3-p12).

Imagen profesional desvirtuada y desconocimiento del rol profesional integrado

«...el gerente de finanzas de acá (...) me decía, qué hacen cosas las enfermeras que yo no tenía idea (...) porque la mayoría de la gente el conocimiento que tienen es como de la enfermería clínica, en la atención directa del paciente, pero todos los temas de gestión no es siempre sabido, hay como todavía mucho mito, hay mucho... desconocimiento al respecto» (E1-p26).

«...es el mito, que es social, que una persona que no conoce qué piensa de una enfermera, que la imagen de la enfermera es con una inyección o tomando la presión ¿0 no?..» (E2-p36).

Facilidades:

Conciencia de las propias capacidades

«...es importante el posicionamiento y también el pelear entre comillas por este posicionamiento ( $\mathrm{N}$ de I: énfasis)... evidenciar cuál es tu aporte y creo que la parte de enfermería en las instituciones de salud tiene que estar presente, ojalá en la mayor parte de las decisiones....tengo la suerte de que participé en muchos comités, participé en el comité científico, en el docente, en el de insumos, en el de farmacia...hay que luchar» (E2-p21).

«... poder ir identificando las distintas personas cuáles son sus fortalezas y en el fondo construir un grupo con eso, (...) te va construyendo un poco el que te vayan viendo como un líder natural...» (E1- p38).

Reconocimiento legal del rol

«...sí (...) el 2013 ...primero con el nombre de enfermera jefe y ahora con el nombre de subgerente de enfermería, pero en general las funciones siempre han sido las mismas, salvo que ...durante el primer periodo estaba a cargo de la dirección médica y en este segundo periodo tengo dependencia directa del gerente general. Esa es como la diferencia de la organización» (E1-p8).

4. El peso de las estructuras organizacionales «tu rol es parte de un hospital». En el estudio, esta categoría tiene dimensiones vinculadas entre sí y organizadas en los conceptos de reconocimiento institucional, visibilidad y autonomía.

Reconocimiento institucional

«...en la medida de que tú, o la parte directiva de las instituciones conocen tu trabajo ahí se valora» (E1-p25). 
«...se llaman acreditaciones institucionales y que estamos partiendo y ahí uno empieza a entender lo importante que es, y en eso la enfermera juega un rol importante (...) entonces cuando uno se mete en este centro te das cuenta de verdad que tu rol va más allá de un servicio, tu rol es parte de un hospital (N de I: énfasis)» (E2-p 2).

Visibilidad

«... la enfermera hoy en día participa de muchos comités en un hospital...» (E2-p3).

«...enfermería a nivel de las instituciones y como estructura obviamente tiene que estar posicionada como está hoy en día...» (E1 p20).

Autonomía

«...la autonomía la tenemos, quizás aparente, para algunas profesiones, pero orgullosamente, yo puedo decir que este hospital se acreditó, gracias al trabajo ciento por ciento de las enfermeras supervisoras de todos los servicios...» (E2-p2).

«... la enfermera en el rol de líder y en el rol autónomo, tenemos que tener claro dónde estamos, para qué estamos y cómo lo hacemos y no al "lote", con un marco protocolizado anterior que nos va regular, en el fondo, lo que hacemos» (E3-p2).

Como una forma de aproximarse a la comprensión del fenómeno liderazgo-autonomía, se presenta en la siguiente tabla, la organización categorial develada en el estudio, que muestra la vinculación entre las dimensiones, según el modelo de liderazgo integral de Ken Wilber ${ }^{28}$ (Tabla 2).

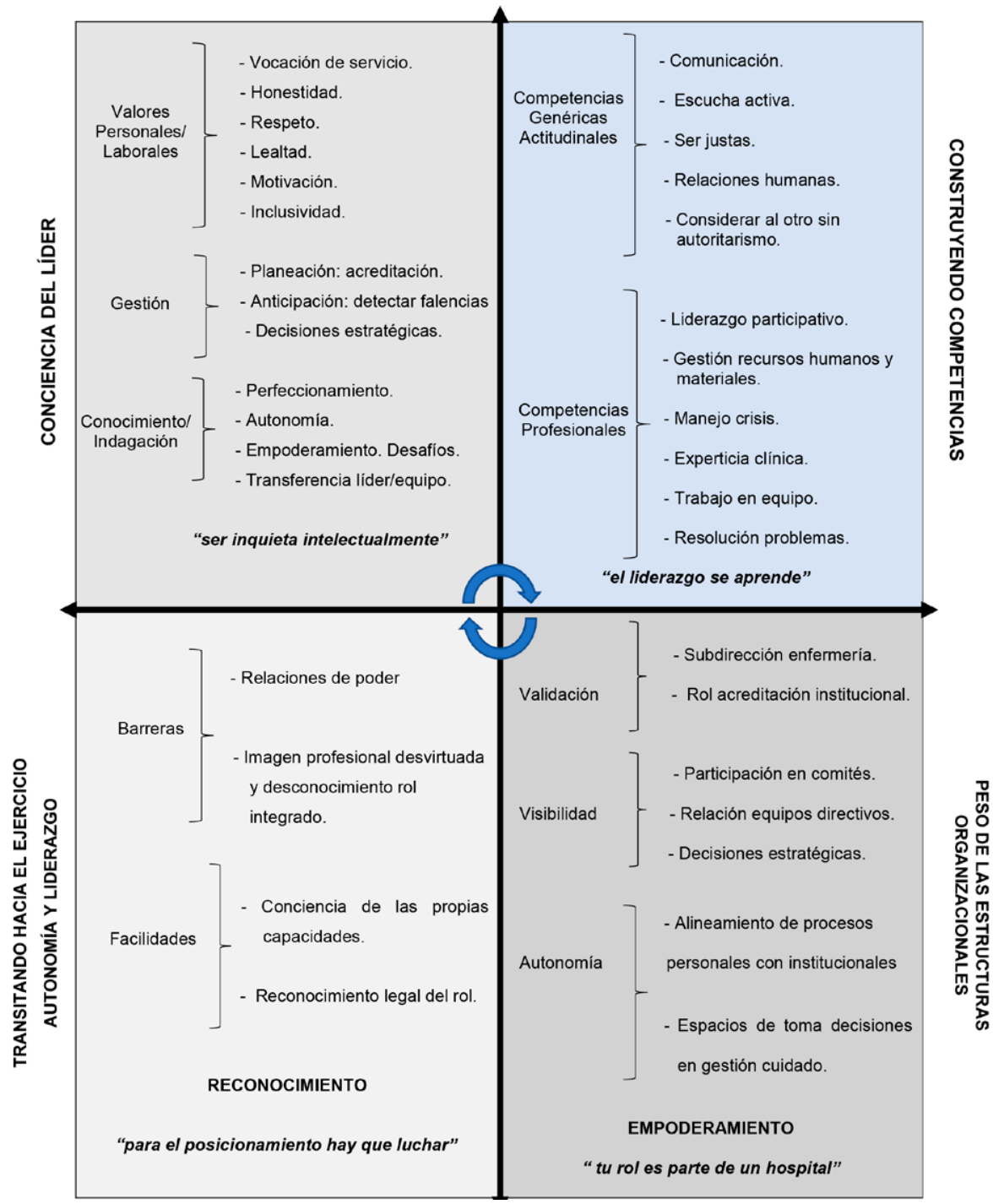

Tabla 2. Categorías develadas en el estudio, según modelo liderazgo integral de Wilber ${ }^{28}$ 


\section{Discusión}

El estudio permitió acercar la realidad subjetiva a una comprensión del fenómeno desde la voz de las propias protagonistas quienes perciben esa realidad. El liderazgo integral considera al líder al interior de una institución, en relación con los grupos y estructuras organizacionales de las cuales depende ${ }^{35}$. Las categorías encontradas fueron «Conciencia del líder ser inquieta...intelectualmente», «Construyendo competencias, el liderazgo se aprende», «Transitando hacia el ejercicio de la autonomía y el liderazgo, para el posicionamiento hay que luchar» $\mathrm{y}$ «El peso de las estructuras organizacionales, tu rol es parte de un hospital»

La primera categoría Conciencia del líder, hace referencia a las intenciones del líder y a la subjetividad de su experiencia, se focaliza en lo que él valora para tomar decisiones de manera autónoma ${ }^{35}$. Los principales atributos según las informantes son los valores personales, se reconoce que estos potencian el desarrollo profesional y el reconocimiento social ${ }^{36,37}$. Esta afirmación proveniente del yo, es invalidada por la visión de los actores sociales, quienes perciben al profesional orientado más bien hacia la realización de actividades fundamentalmente técnicas, en un papel de sumisión a otras disciplinas, por lo cual queda demostrado tanto en los discursos del presente estudio, como en investigaciones revisadas ${ }^{38,39}$.

Sin embargo, otros estudios reconocen a las competencias técnico-operativas para la gestión y administración, como elementos constitutivos del proceso de trabajo de enfermeras/os, brindándole un carácter autónomo y distintivo del resto de los profesionales, situación que representaría un potencial para el reconocimiento social ${ }^{40-42}$. Ambas perspectivas reflejan la ambigüedad existente que limita el genuino reconocimiento social del profesional de enfermería, en su rol particular y en el ejercicio del liderazgo y autonomía.

En cuanto a la gestión, los discursos pusieron de manifiesto la capacidad de planeación a partir del análisis de falencias, para tomar decisiones estratégicas. Se identifican coincidencias con un estudio realizado en México, referido al modelo de dirección por enfermeras en hospitales, en el cual se establece que para el ejercicio del liderazgo y la autonomía profesional, es necesario tener/disponer de competencias relacionadas con el pensamiento estratégico y la proactividad frente a situaciones críticas, necesarias para que el profesional se instale no sólo en la microgestión clínica, sino que avance al nivel de la macrogestión de políticas en salud, como una forma de tener una mayor visibilidad ${ }^{43}$.

Para el logro de las competencias en el área de la gestión las enfermeras entrevistadas pusieron en relieve el ser inquieta...intelectualmente, aspecto reflejado en la necesidad de acceder al conocimiento y a tomar decisiones en base a la indagación, ambos descritos como aspectos facilitadores para el liderazgo, ya que permiten generar innovaciones en la gestión y mantener la motivación, la satisfacción laboral, además de lograr la adhesividad al modelo institucional ${ }^{44,45}$.

La categoría Construyendo competencias, está enfocada fundamentalmente, a cómo se construye el liderazgo y la autonomía desde el yo individual, pero ahora puesto en acción o a través de un desempeño determinado con los demás. Se identificaron dos grandes áreas competenciales, genérica actitudinal y profesional, ambas en una ola recursiva y dinámica que vincula esta categoría con la de conciencia del líder según el modelo de Wilber ${ }^{28,35}$.

Las competencias genéricas actitudinales reveladas en los relatos de las entrevistadas corresponden a los aspectos comunicacionales en la relación entre las personas, se considera al otro sin autoritarismo, estas se vinculan con las competencias profesionales que se manifiestan en un liderazgo participativo y el manejo de situaciones críticas.

Al vincular las categorías «conciencia del líder» con la de «construyendo competencias», el liderazgo es entendido por quienes lo ejercen, como un proceso de influencia grupal, que puede tener un carácter de conquista personal, a través de la reflexión acerca de las propias prácticas para la detección de brechas de conocimiento y la motivación para acceder a la educación continua, además de fortalecer las competencias del líder. Este proceso quedó reflejado en la etiqueta de la categoría analizada «el liderazgo se aprende» ${ }^{44}$. 
La categoría «Transitando hacia el ejercicio de la autonomía y el liderazgo», según el modelo de Wilber se reconoce la naturaleza de los códigos, reglas, prejuicios construidos intersubjetivamente y que determinan la conducta del líder ${ }^{28,35}$.

Se identificaron barreras y facilidades para el reconocimiento del líder, en una lucha para ubicarse en una posición reconocida. Las barreras se encontraron, principalmente, en la existencia de relaciones de poder al interior de las instituciones y una imagen profesional desvirtuada, por el desconocimiento del rol profesional integrado por parte del equipo de salud. En general, en todas las instituciones sociales prevalece una relación entre poder y saber, sin embargo, actualmente, el conocimiento ya no es privativo de algunos profesionales, ya que es compartido por otros actores, quienes van asumiendo de manera progresiva nuevos roles de liderazgo y de poder en las organizaciones. El ejercicio del poder en las instituciones de salud, limita el pleno posicionamiento del liderazgo de los profesionales de enfermería, lo que condiciona no sólo la imagen proyectada, sino la percepción que se tiene de sí mismo ${ }^{38,39}$.

En cuanto a las facilidades, las enfermeras relatan que estas se basan en la conciencia de sus propias capacidades para mejorar la imagen y lograr la validación social, lo cual se refleja claramente en la referencia que hace una de las entrevistadas "para el reconocimiento hay que luchar". Junto con ello le asignan valor al reconocimiento del rol profesional en la legislación chilena ${ }^{5-7}$. Si bien es cierto, las enfermeras entrevistadas comprenden la importancia del conocimiento, para lograr el empoderamiento como líderes, ellas no realizan un análisis crítico del ejercicio del poder en lo cotidiano de las instituciones. Toda vez, que la existencia de grupos de poder al interior de los centros de salud, no sólo repercute en el posicionamiento del líder, sino que muchas veces en la calidad de los cuidados ${ }^{19}$.

En la última categoría «El peso de las estructuras organizacionales». permite comprender el significado que tiene el lugar de trabajo para el líder, como dimensiones exteriores evidenciadas a través de las relaciones jerárquicas institucionales, políticas, normativas, protocolos, planes estratégicos, que permiten un alto nivel de autonomía del personal y que son reflejo de la organización ${ }^{35}$.

Los resultados del estudio ponen de manifiesto la forma en que las estructuras institucionales se vinculan con el profesional y se convierten en un apoyo para el empoderamiento del liderazgo y del ejercicio de la autonomía. La autonomía se expresa en la manera, que las profesionales entrevistadas alinean sus procesos personales con los institucionales, comprometerse y representar a la organización en correspondencia, situación simbolizada en la frase de una de las enfermeras "tu rol es parte de un hospital" 45, 46 .

Las informantes señalan la existencia de reconocimiento institucional del liderazgo y autonomía, con base en la instalación de enfermeras/os en las estructuras de gestión, así como, en la forma de subdirecciones de gestión del cuidado. Además, se les reconoce por el papel que les ha correspondido jugar en los procesos de acreditación de los centros de salud, los cuales deben cumplir un conjunto de estándares de calidad normados por el Ministerio de Salud de Chile.

\section{Conclusiones}

El estudio permitió identificar las competencias del líder al priorizar los valores personales y la búsqueda del conocimiento para la gestión, por sobre aspectos técnicos para potenciar el liderazgo y la autonomía, sin embargo, esta visión no se ajusta a la de otros actores sociales, quienes perciben al profesional más bien orientado hacia la realización de actividades fundamentalmente técnicas, en un papel de sumisión a otras disciplinas.

En el plano directivo institucional existe un reconocimiento de los profesionales de enfermería que facilitan asumir el liderazgo y la autonomía, pero en el plano de los equipos de salud se perciben barreras generadas, fundamentalmente, por la persistencia de un modelo basado en decisiones médicas y por el ejercicio del poder, lo que limitaría la máxima expresión del liderazgo y de la autonomía, además de impedir la visibilidad del particular rol de los profesionales de enfermería.

Los profesionales identifican que dentro de las facilidades está la conciencia de sus propias capacidades y el reconocimiento del rol profesional en la legislación chilena, así como de las propias instituciones 
en las que laboran, lo cual brindaría un sustento para potenciar la capacidad de empoderamiento como lideres que ejercen su autonomía.

El estudio logra establecer que el líder se mueve en un escenario dual y complejo, de menor reconocimiento por parte de algunos integrantes del equipo de salud, que incluye a sus pares, así como usuarios y un creciente empoderamiento personal de su rol autónomo.

Estos hallazgos deben impulsar al colectivo de enfermeras/os, para incrementar la visibilización del rol, a través de la participación en los niveles de alta dirección y en instancias colegiadas gremiales de la enfermería, debido a que esos espacios colectivos y decisionales potencian el reconocimiento y el empoderamiento del profesional.

\section{Limitaciones}

Los resultados de este estudio se refieren sólo a la perspectiva de las enfermeras seleccionadas que ejercían cargos de jefaturas, en centros de atención cerrada de la región, por lo que es importante escuchar otras voces en futuras investigaciones, como la percepción de enfermeros, directivos, pares o subalternos, para así ampliar la comprensión del desarrollo y ejercicio de la autonomía y el liderazgo en enfermería.

El estudio nos transportó a otras áreas complejas, como es el ejercicio del poder, la identidad profesional, la imagen social, los cuales, aunque son relevantes, se alejaban de los objetivos propuestos, por lo que queda abierta la posibilidad para ser analizadas en futuros abordajes.

\section{Responsabilidades éticas}

Protección de personas y animales. Los autores declaran que en este estudio no se realizaron experimentaciones en seres humanos ni animales.

Confidencialidad de datos. Los autores declaran que en el artículo no aparecen datos de pacientes.

Derecho a la privacidad y consentimiento informado. Los autores han obtenido el consentimiento informado de los sujetos referidos en el artículo. Este documento está en poder del autor de correspondencia. Conflicto de interés. Los autores declaran no tener conflictos de interés.

Fuentes de financiamiento. Ninguno.

Agradecimientos. A las colegas que participaron en el estudio, que compartieron sus experiencias vitales como líderes.

\section{Referencias}

1. Guerrero-Nuñez S, Cid-Henríquez P. Una reflexión sobre la autonomía y el liderazgo en enfermería. Aquichan. 2015; 15(1): 129-40. http://dx.doi.org/10.5294/aqui.2015.15.1.12

2. Martínez-Martín ML, Chamorro-Rebollo E. Historia de la Enfermería, Evolución histórica del cuidado enfermero. $2^{a}$ ed. Barcelona: Elsevier; 2011.

3. Dandicourt-Thomas C, Hernández-Valdez E, Espinoza-Moreno TM, Pérez-Siguas RE. Pasado y presente de la función enfermera en el acto del cuidado. Health Care \& Global Health. 2017; 1(1): 49-54. http://dx.doi.org/10.22258/hgh.2017.11

4. Letelier-Valdivia M, Velandia-Mora, AL. Profesionalismo en enfermería, el hábito de la excelencia del cuidado. Av.enferm. 2010; 28(2): 145-58.

5. Ministerio de Salud. Artículo 113 de la Ley 19536 Concede una bonificación extraordinaria para enfermeras y matronas que se desempeñan en condiciones que indica, en los establecimientos de los servicios de salud. Santiago, Chile: MINSAL; 1997.

6. Ministerio de Salud. Norma General Administrativa No 19. "Gestión del cuidado de enfermería para la atención cerrada”. Santiago, Chile: MINSAL; 2007.

7. Milos P, Bórquez B, Larraín AI. La gestión del cuidado en la legislación chilena: Interpretación y alcance. Cienc. enferm. 2010; 16(1): 17-29. 
8. Poblete-Troncoso M, Valenzuela-Suazo, S. Cuidado humanizado: un desafío para las enfermeras en los servicios hospitalarios. Acta Paul. Enferm. 2007; 20(4): 499-503.

9. Souza-Souza LP, Ferreira-Cordeiro AL, Nunes-de Aguiar R, De Freitas-Santana JM, Veloso-Dias O, Vieira MA, et al. El liderazgo en la visión de enfermeros líderes. Enfermería glob. 2013; 12(2):26880. http://dx.doi.org/10.6018/eglobal.12.2.154801

10. Luengo-Martínez C, Paravic-Klijn T, Burgos-Moreno M. Profesionalismo en enfermería: una revisión de la literatura. Enferm. univ. 2017; 14(2): 131-42. https://doi.org/10.1016/j.reu.2017.02.001

11. Luengo-Martínez C, Paravic-Klijn T. Autonomía profesional: factor clave para el ejercicio de la Enfermería Basada en la Evidencia. Index enferm. 2016; 25(1-2): 42-6.

12. Medina-Moya JL, Schubert-Backes VM, do Prado ML, Sandin MP. La enfermería, como grupo oprimido: las voces de las protagonistas. Texto contexto enferm. 2010; 19(4): 609-17. http://dx.doi.org/10.1590/S0104-07072010000400002

13. Scully NJ. Leadership in nursing: The importance of recognizing inherent values and attributes to secure a positive future for the profession. Collegian ACN. 2015; 22 (4): 439-44. https://doi.org/10.1016/j.colegn.2014.09.004

14. Yáñez-Gallardo RJ, y Valenzuela-Suazo S. Conductas crítica para experimentar confianza en el liderazgo en enfermería en un hospital de alta complejidad. Aquichan.2013; 13(2): 186-96. https://doi.org/10.5294/aqui.2013.13.2.4

15. Marcellino de Melo-Lanzoni G, Schlindwein-Meirelles BH. Liderazgo del enfermero: una revisión integradora de la literatura. Rev Lat.-Am Enferm. 2011;19(3): 09 pantallas 651-8. http://dx.doi.org/10.1590/S0104-11692011000300026

16. De Lima-Trindade L, Coelho-Amestoy S, Adyles-Muniz L, Biolchi T, Pires de Pires DE, SchubertBackes VM. Influencia de los estilos de liderazgo del enfermero en las relaciones interpersonales del equipo de enfermería. Enfermería glob. 2011; 10(22): 09 pantallas.

17. González-Álvarez L, Guevara-Sotomayor E, Morales-Figueroa G, Segura-Hernández P, LuengoMartínez C. Relación de la satisfacción laboral con estilos de liderazgo en enfermeros de hospitales públicos, Santiago, Chile. Cienc. enferm. 2013; 19(1): 11-21.

http://dx.doi.org/10.4067/S0717-95532013000100002

18. Garlet ER, de Lima-Trindade L, Dias-da Silva Lima MA, de Lourenzi-Bonilha AL. The resignification of management processes in care procedures in nursing. Online Braz. J. Nurs. 2006; 5(3).

19. Tapp D, Stansfield K, Stewart J. La autonomía en la práctica de enfermería. Aquichan. 2005; 5(1): $114-27$.

20. Quezada-Torres C, Illesca-Pretty M, Cabezas-González M. Percepción del liderazgo en las(os) enfermeras(os) de un hospital del sur de Chile. Cienc. enferm. 2014; 20(2): 41-51. http://dx.doi.org/10.4067/S0717-95532014000200005

21. Drucker PF. El ejecutivo eficaz. México: Debolsillo; 2012.

22. Cortés-González J, Hernández-Saavedra MP, Marchena-Rivera TG, Marqueti-Machado ML, Nava-Galán MG. Estilos de liderazgo en jefes de Servicio de Enfermería. Rev. enf. neurol. 2012; 12(2): 84-94.

23. Cárcamo-Fuentes C, Rivas-Riveros E. Estilo de liderazgo en profesionales de enfermería según su función en los sectores público o privado en Temuco, Chile, 2015. Aquichan, 2017; 17(1): 70-83. http://dx.doi.org/10.5294/aqui.2017.17.1.7

24. Pazetto-Balsanelli A, Kowal-Olm Cunha IC. Liderazgo en enfermería en las unidades de cuidados intensivos y su relación con el entorno laboral. Rev. Lat.-Am. Enferm. 2015; 23(1): 106-13. http://dx.doi.org/10.1590/0104-1169.0150.2531

25. Sánchez-Montalván SE, Vaca-Aguirre I, Padilla-Sánchez JP, Quezada-Condolo CR. Teorías de liderazgo contemporáneo: análisis de la dinámica relacional. Oikos 2014; 18(37): 41-61.

26. Rozo-Mogollón SM, Abaunza-de González, M. Liderazgo transaccional y transformacional. Av. enferm. 2010; 28(2): 62-72. 
27. Valderrama-Sanabria ML. Experiencias exitosas de liderazgo de enfermería. Rev. cuid. 2014; 5(2): 765-73. http://dx.doi.org/10.15649/cuidarte.v5i2.89

28. Wilber K. Una teoría de todo: Una visión integral de la ciencia, la política, la empresa y la espiritualidad. Barcelona: Kairós; 2001.

29. Husserl E. La idea de la fenomenología: Cinco Lecciones, trad. de Miguel García-Baró. México: FCE. 1982.

30. Martínez-Salgado C. El muestreo en investigación cualitativa: Principios básicos y algunas controversias. Ciên Saúde Colet. 2012; 17 (3): 613-9.

31. Noreña AL, Alcaraz-Moreno N, Rojas JG, Rebolledo-Malpica D. Aplicabilidad de los criterios de rigor y éticos en la investigación cualitativa. Aquichan. 2012; 12(3):263-74. http://www.redalyc.org/articulo.oa ?id=741249480006

32. De Cássia Rocha-Moreira, R., Mendonça-Lopes RL, De Andrade-Santos N. Entrevista fenomenológica: peculiaridades para la producción científica en enfermería. Index enferm. 2013; 22(1-2): 107-10. http://dx.doi.org/10.4321/S1132-12962013000100024

33. Sayago $\mathrm{S}$. El análisis del discurso como técnica de investigación cualitativa y cuantitativa en ciencias sociales. Cinta moebio. 2014; (49):1-10. http://dx.doi.org/10.4067/S07 17-554X2014000100001

34. Taylor SJ, Bogdan R. Introducción a los métodos cualitativos de investigación: la búsqueda de significados. Barcelona: Paidós; 1987.

35. Reyes-Jácome L, Lara-Rodríguez G. El liderazgo integral en las organizaciones. Av Psicol Latinoam. 2011; 29(1): 161-75.

36. Campos-Pavone Zoboli EL, Cabral-Schveitzer M. Valores de la enfermería como práctica social: una metasíntesis cualitativa. Rev. Lat.-Am. Enferm. 2013; 21 (3):09 pantallas. http://dx.doi.org/10.1590/S0104-11692013000300007

37. Stanley D. Congruent leadership: values in action. J Nurs Manag. 2008; 16(5): 519-24. http://dx.doi.org/10.1111/j.1365-2834.2008.00895.x

38. Kappes-Ojeda C, Mella-Moraga R. El ejercicio del poder y su relación con el rol profesional de un grupo de enfermeras en la gestión de los cuidados. Cuid. enferm. educ. salud. 2014; 1(1): 52-68.

39. Amezcua M. Foucault y las enfermeras: pulsando el poder en lo cotidiano. Index enferm. 2009; 18(2): 77-9.

40. Bustamante-Ubilla MA, Grandón-Avendaño ML, Lapo-Maza MC. Caracterización del clima organizacional en hospitales de alta complejidad en Chile. Estud. Gerenc. 2015; 31(137):432-40. https://doi.org/10.1016/j.estger.2015.08.003

41. Soto-Fuentes P, Reynaldos-Grandón K, Martínez-Santana D, Jerez-Yañez O. Competencias para la enfermera/o en el ámbito de gestión y administración: desafíos actuales de la profesión. Aquichan. 2014; 14(1): 79-99.

42. Curtis EA, de Vries J, Sheerin FK. Developing leadership in nursing: exploring core factors. Br J Nurs. 2011; 20(5): 306-9. http://dx.doi.org/10.12968/bjon.2011.20.5.306

43. Gómez-Torres D, Ángeles-Ávila G, Martínez-Garduño MD. Modelo de dirección de hospitales por enfermeras. Aquichan. 2011; 11(3): 316-26.

44. Costa-Mendes IA, Arena-Ventura CA, Trevizan MA, Marchi-Alves LM, Duarte de Souza-Junior V. Educación liderazgo y colaboraciones: potencialidades que la Enfermería puede dar a la Cobertura Universal en Salud. Rev. Lat.-Am. Enferm. 2016; 24: e2673. http://dx.doi.org/10.1590/1518-8345.1092.2673

45. Dentico JP. Going nowhere fast? Simulations and the future of leadership development. 2009 August 01. En: Integral Leadership Review. Disponible en: https://bit.ly/2uUjN9S

46. Cherian S, Karkada S. A review on leadership in nursing. Int. j. nurs. res. pract. 2017; 4(1): 58-66. https://doi.org/10.15509/IJNRP.2017.4.1.347 